\title{
Control of hysteresis in smart actuators with application to micro-positioning
}

\author{
Xiaobo Tan*, John S. Baras, P.S. Krishnaprasad \\ Institute for Systems Research and Department of Electrical and Computer Engineering, University of Maryland, \\ College Park, MD 20742, USA
}

Received 15 September 2003; received in revised form 30 September 2004; accepted 30 September 2004

Available online 11 November 2004

\begin{abstract}
Hysteresis in smart material actuators makes the effective use of these actuators quite challenging. The Preisach operator has been widely used to model smart material hysteresis. Motivated by positioning applications of smart actuators, this paper addresses the value inversion problem for a class of discretized Preisach operators, i.e., to find an optimal input trajectory given a desired output value. This problem is solved through optimal state transition of a finite state machine (FSM) that corresponds to the discretized Preisach operator. A state-space reduction scheme for the FSM is developed, which significantly saves the memory and the computation time. Experimental results on micro-positioning control of a magnetostrictive actuator are presented to demonstrate the effectiveness of the proposed approach.
\end{abstract}

(C) 2004 Elsevier B.V. All rights reserved.

Keywords: Preisach operator; Inversion; Hysteresis; Magnetostriction; Micro-positioning control

\section{Introduction}

Smart materials, such as magnetostrictives, piezoelectrics, electroactive polymers (EAPs) and shape memory alloys (SMAs), all display certain coupling phenomena between applied electro-magnetic/thermal

\footnotetext{
* Corresponding author. Present address: Department of Electrical and Computer Engineering, Michigan State University, East Lansing, MI 48824, USA. Tel.: +15174325671; fax: +15173531980 .

E-mail addresses: xbtan@msu.edu (X. Tan), baras@isr. umd.edu (J.S. Baras), krishna@isr.umd.edu (P.S. Krishnaprasad).
}

fields and their mechanical properties. Actuators and sensors made of such materials, often called smart actuators and smart sensors, have been receiving tremendous interest in the past two decades, due to their broad applications in areas of aerospace, manufacturing, defense, and civil infrastructure systems. However, the hysteretic behavior widely existing in these materials makes their effective use quite challenging [12].

A fundamental idea in coping with hysteresis is to formulate the mathematical model of hysteresis and use inverse compensation to cancel out the hysteretic effect, see, e.g., [10,20,16,6,19]. Hysteresis models can be roughly classified into physics-based models 
and phenomenological models. The most popular phenomenological hysteresis model used for smart materials has been the Preisach operator $[1,10,15,7,8,19,13,5]$. A similar type of operator, called Krasnosel'skii-Pokrovskii (KP) operator has also been used $[2,6]$.

The inverse compensation approach mentioned above is concerned with the trajectory inversion problem: given a desired output trajectory, one computes the corresponding input trajectory whose output trajectory matches the desired one. In many applications like micro-positioning, one might be more interested in the following problem: given a desired output value, find the input trajectory such that the final value of the output matches the desired value. To distinguish this problem from trajectory inversion, it is called the value inversion problem in this paper. Such problems have been well studied for linear systems (see, e.g., [14] and the references therein), but to the authors' best knowledge, very little has been done in the context of hysteretic systems.

In this paper the value inversion problem for a class of discretized Preisach operators is formulated and solved. Such an operator is represented as a finite state machine (FSM), and the value inversion problem is transformed into a reachability problem for the FSM. The hysteretic dynamics of the FSM is fully characterized, based on which its reachability is proved. Construction of the input sequence for a given state transition is described through an example. Having observed that in practice there may exist a large number of equivalent states for the FSM, we propose a state space reduction scheme, which significantly saves the storage space and the computation time. An algorithm for generating the optimal (the sense of "optimality" will be clear later) representative state in each equivalence class is presented. Experimental results on micro-positioning control of a magnetostrictive actuator are used to demonstrate the effectiveness of the proposed approach.

The remainder of the paper is organized as follows. The Preisach operator is briefly reviewed and a discretization scheme is introduced in Section 2. Section 3 studies the state reachability problem for the FSM. Section 4 is devoted to the state-space reduction scheme. Experimental results are reported in Section 5. Finally, concluding remarks are provided in Section 6.

\section{The Preisach operator and its discretization}

\subsection{Review of the Preisach operator}

For a detailed treatment on the Preisach operator, one is referred to $[11,22,4]$. For a pair of thresholds $(\beta, \alpha)$ with $\beta \leqslant \alpha$, consider a simple hysteretic element $\hat{\gamma}_{\beta, \alpha}[\cdot, \cdot]$, as illustrated in Fig. 1. For $u \in C([0, T])$ (the space of continuous functions on $[0, T])$ and an initial configuration $\zeta \in\{-1,1\}, \omega=\hat{\gamma}_{\beta, \alpha}[u, \zeta]$ is defined as, for $t \in[0, T]$,

$\omega(t) \triangleq \begin{cases}-1 & \text { if } u(t)<\beta, \\ 1 & \text { if } u(t)>\alpha, \\ \omega\left(t^{-}\right) & \text {if } \beta \leqslant u(t) \leqslant \alpha,\end{cases}$

where $\omega\left(0^{-}\right)=\zeta$ and $t^{-} \triangleq \lim _{\varepsilon>0, \varepsilon \rightarrow 0} t-\varepsilon$.

The operator $\hat{\gamma}_{\beta, \alpha}$ is often referred to as a Preisach hysteron (called hysteron hereafter). Define the Preisach plane

$\mathscr{P}_{0} \triangleq\left\{(\beta, \alpha) \in \mathbb{R}^{2}: \beta \leqslant \alpha\right\}$,

where $(\beta, \alpha) \in \mathscr{P}_{0}$ is identified with $\hat{\gamma}_{\beta, \alpha}$. For $u \in$ $C([0, T])$ and a Borel measurable configuration $\zeta_{0}$ of all hysterons, $\zeta_{0}: \mathscr{P}_{0} \rightarrow\{-1,1\}$, the output of the Preisach operator $\Gamma$ is defined as

$\Gamma\left[u, \zeta_{0}\right](t)=\int_{\mathscr{P}_{0}} \mu(\beta, \alpha) \hat{\gamma}_{\beta, \alpha}\left[u, \zeta_{0}(\beta, \alpha)\right](t) \mathrm{d} \beta \mathrm{d} \alpha$,

where the weighting function $\mu(\beta, \alpha)$ is called the Preisach density function. It is assumed in this paper that $\mu$ has a compact support

$\mathscr{P} \triangleq\left\{(\beta, \alpha) \in \mathscr{P}_{0} \mid \beta \geqslant \beta_{0}, \alpha \leqslant \alpha_{0}\right\}$,

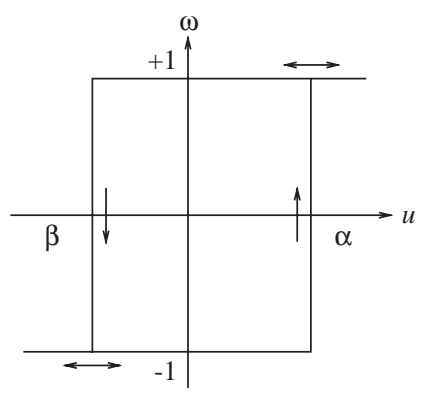

Fig. 1. A Preisach hysteron $\hat{\gamma}_{\beta, \alpha}[\cdot, \cdot]$. 

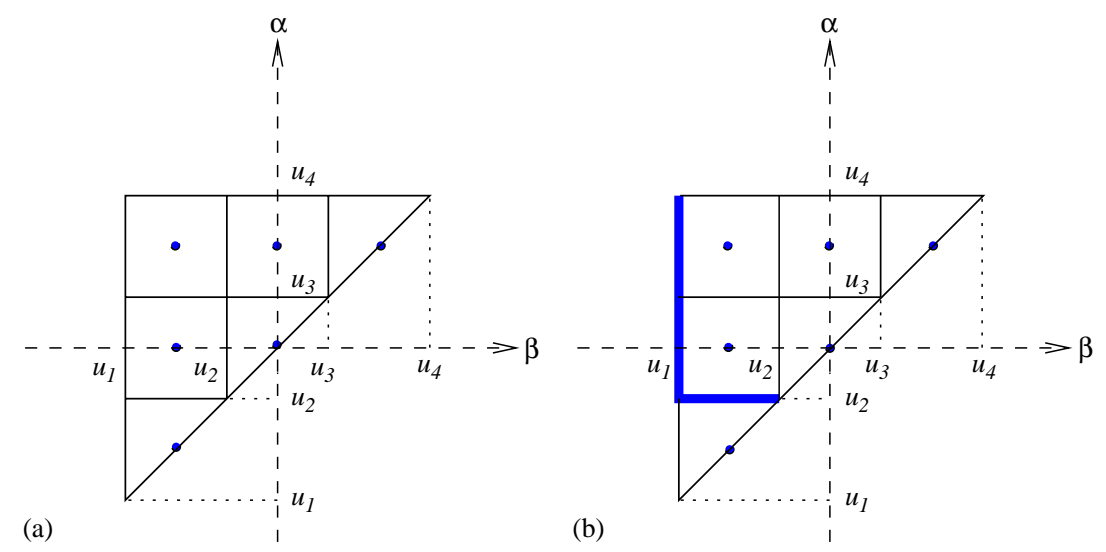

Fig. 2. (a) Discretization of the Preisach plane ( $L=3$ ); (b) memory curve "001" (bold-faced lines).

for some $\beta_{0}, \alpha_{0} . \mathscr{P}$ is also called the Preisach plane when no confusion arises.

At any time $t, \mathscr{P}$ is a disjoint union of two sets, $\mathscr{P}_{+}(t)$ and $\mathscr{P}_{-}(t)$, where $\mathscr{P}_{+}(t)\left(\mathscr{P}_{-}(t)\right.$, resp. $)$ consists of points $(\beta, \alpha)$ such that the output of $\hat{\gamma}_{\beta, \alpha}$ at $t$ is +1 ( -1 , resp.). Under mild conditions, each of $\mathscr{P}_{+}(t)$ and $\mathscr{P}_{-}(t)$ is a connected set, and the boundary between them, called the memory curve, characterizes the memory of the Preisach operator. The memory curve has a staircase structure and the coordinates of its intersection with the line $\alpha=\beta$ correspond to the current value of the input. The set of all memory curves is denoted as $\Psi$. The memory curve $\psi_{0}$ at $t=0$ is called the initial memory curve and hereafter it will be put as the second argument of $\Gamma$.

\subsection{The discretized Preisach operator}

In identification of the Preisach density function a discretization step is involved in one way or another (see [17] for a review of identification methods). A natural discretization scheme is as follows.

Considering the operating limits of actuators, we assume the input range to be $\left[u_{\min }, u_{\max }\right]$. This range is uniformly discretized into $L+1$ levels. The set of input levels is denoted as $U \triangleq\left\{u_{l}, 1 \leqslant l \leqslant L+1\right\}$ with $u_{l}=u_{\min }+(l-1) \delta_{u}$, where $\delta_{u}=\left(u_{\max }-u_{\min }\right) / L$. $L$ will be called the discretization level. Input discretization leads to discretization of the Preisach plane. Fig. 2(a) shows the discretization scheme for $L=3$. The density distribution inside each cell is assumed to

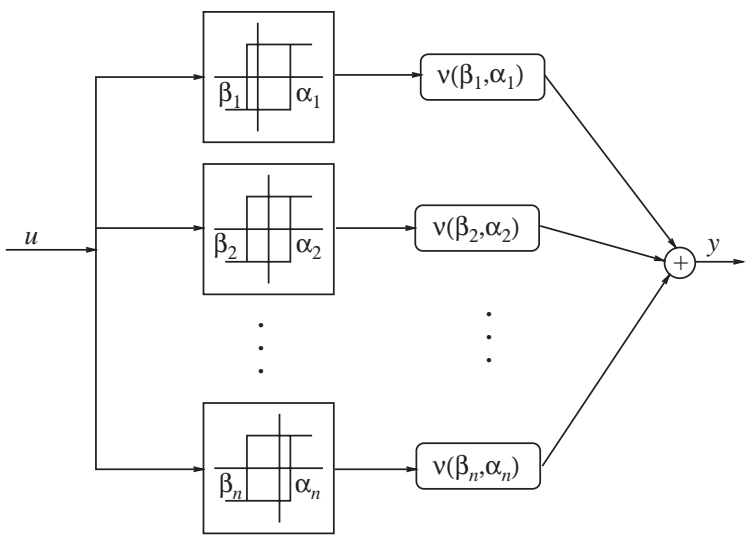

Fig. 3. The discretized Preisach operator.

concentrate at the cell center (represented by dark dots in Fig. 2(a)) and this results in a discretized Preisach operator, which is now a weighted sum of $L(L+1) / 2$ hysterons (see Fig. 3). In Fig. 3, $v\left(\beta_{i}, \alpha_{i}\right)$ denotes the weight for the hysteron $\hat{\gamma}_{\beta_{i}, \alpha_{i}}$. Note that although uniform discretization is considered here, the results presented in this paper apply directly to the case of non-uniform discretization.

\section{The value inversion problem}

\subsection{Formulation of the value inversion problem}

Since the Preisach operator is rate-independent [11], and at any time $t$ the memory curve (and thus the output value) depends only on the dominant maximum 
and minimum values in the past input [11], we restrict ourselves to the discrete time setting and put a sequence instead of a continuous time function as the first argument of $\Gamma$.

Let $S$ be the set of input strings taking values in $U=\left\{u_{l}, 1 \leqslant l \leqslant L+1\right\}$, i.e., if $s \in S$ is a string of length $n$, then $s[i] \in U, 1 \leqslant i \leqslant n$. To avoid ambiguity, it is tacitly understood that the input is changed monotonically from $s[i]$ to $s[i+1]$. Define $S_{\mathrm{A}}$ to be the set of alternating input strings [4], in the sense that, if $s_{\mathrm{a}} \in S_{\mathrm{A}}$, then $\left(s_{\mathrm{a}}[i+2]-s_{\mathrm{a}}[i+1]\right)\left(s_{\mathrm{a}}[i+1]-s_{\mathrm{a}}[i]\right)<0, \forall i>0$.

In micro-positioning, one is mainly interested in the final position and cares less about the transient trajectory. This motivates us to study the value inversion problem for the (discretized) Preisach operator. Let $\Psi_{\mathrm{d}}$ denote the set of memory curves for the discretized Preisach operator. The value inversion problem is formulated as: given a desired output value $\bar{y}$ and an initial memory curve $\psi_{0} \in \Psi_{\mathrm{d}}$, find $s_{\mathrm{a}}^{*} \in S_{\mathrm{A}}$, such that

$\left|\Gamma_{\mathrm{f}}\left[s_{\mathrm{a}}^{*}, \psi_{0}\right]-\bar{y}\right|=\min _{s_{\mathrm{a}} \in S_{\mathrm{A}}}\left|\Gamma_{\mathrm{f}}\left[s_{\mathrm{a}}, \psi_{0}\right]-\bar{y}\right|$,

where $\Gamma_{\mathrm{f}}\left[s, \psi_{0}\right]$ denotes the final value of the output of the Preisach operator under input sequence $s$. If there is more than one such string achieving (2), find the one of the minimum length.

Remarks. (1) A discretized Preisach operator is not "onto" since its output takes values in a finite set. Therefore, perfect match is not sought in the formulation above.

(2) Any $s \in S$ can be reduced to some $s_{\mathrm{a}} \in S_{\mathrm{A}}$ using the following rules, starting from $i=1$ : if $(s[i+$ $1]-s[i])(s[i+2]-s[i+1]) \geqslant 0$, delete $s[i+1]$ and re-index. For example, $s=\left(u_{1}, u_{3}, u_{3}, u_{5}, u_{4}, u_{2}\right) \in S$ can be reduced to $s_{\mathrm{a}}=\left(u_{1}, u_{5}, u_{2}\right) \in S_{\mathrm{A}}$. The final values of the output under $s$ and $s_{\mathrm{a}}$ are identical (easy to verify). Hence one only needs to search the optimal input sequence in $S_{\mathrm{A}}$.

(3) The length of an alternating input string is directly linked to the number of input reversals and thus the complexity of implementing that input. Therefore, one seeks $s_{\mathrm{a}}^{*}$ of the minimum length.

\subsection{The state reachability problem}

The discretized Preisach operator is an FSM. Since there are $L(L+1) / 2$ hysterons in a discretized Preisach model with discretization level $L$ and each hysteron takes value in $\{-1,1\}$, the number of states appears to be $2^{L(L+1) / 2}$. This is not the case in general, recalling that each of $\mathscr{P}_{-}$and $\mathscr{P}_{+}$is a connected set (refer to Section 2) and the true state is the memory curve.

Proposition 3.1. For a discretized Preisach operator with discretization level $L$, the number of states is $2^{L}$.

Proof. For a discretized Preisach operator, each memory curve consists of $L$ horizontal or vertical segments of length $\delta_{u}$, so the total number of memory curves is $2^{L}$.

The proof motivates an indexing scheme for the memory curve. Starting from the upper-left corner, we number each memory curve with $L$ bits corresponding to the $L$ segments: 0 represents vertical, and 1 represents horizontal. For instance, the memory curve represented by the bold-faced lines in Fig. 2(b) reads " 001 ". To fix the ordering of bits, we refer to the leftmost (rightmost, resp.) bit as bit $L$ (bit 1, resp.).

A complete description for the FSM can now be given. It has state space

$$
\begin{aligned}
& \Psi_{\mathrm{d}}=\left\{\psi: \psi=\left(\alpha^{L}, \alpha^{L-1}, \ldots, \alpha^{1}\right),\right. \\
& \left.\alpha^{j} \in\{0,1\}, j=1, \ldots, L\right\}
\end{aligned}
$$

and input space $U$. It is a state output automaton [3] since the output $y$ of the Preisach operator depends only on the memory curve $\psi$. Therefore, the value inversion problem is solved if any state of the FSM is reachable, because then all one has to do is to find the state whose corresponding output is closest to the desired value $\bar{y}$.

A state-space representation of a general Preisach operator can be found in [9] and it is shown there that the state space is approximately reachable. This "approximate reachability" result was also stated in $[11,22]$ (in a more casual way). As one shall see next, the hysteretic dynamics of a discretized Preisach operator can be characterized elegantly in terms of the FSM. The reachability of the FSM then follows from the characterization.

The state transition function $\Xi_{\mathrm{d}}: \Psi_{\mathrm{d}} \times U \rightarrow \Psi_{\mathrm{d}}$ for the FSM can be best described in terms of two state operations, INC: $\Psi_{\mathrm{d}} \rightarrow \Psi_{\mathrm{d}}$ and DEC: $\Psi_{\mathrm{d}} \rightarrow \Psi_{\mathrm{d}}$. For any state $\psi \in \Psi_{\mathrm{d}}$, one can immediately determine 


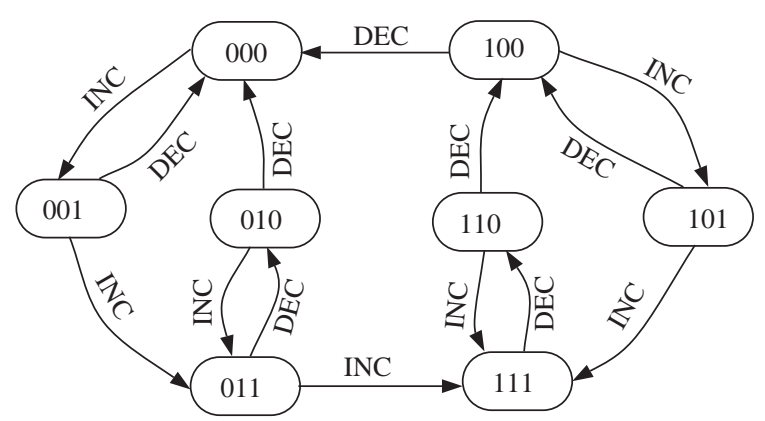

Fig. 4. Operations INC and DEC for $L=3$.

the current input $\tilde{u}(\psi): \tilde{u}(\psi)=u_{n+1}$ if $\psi$ contains $n$ "1"s. For $\psi \in \Psi_{\mathrm{d}}$, define

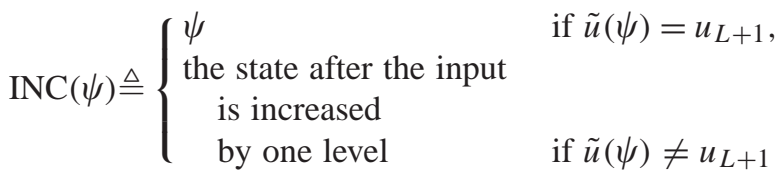
and

$\operatorname{DEC}(\psi) \triangleq \begin{cases}\psi & \text { if } \tilde{u}(\psi)=u_{1}, \\ \begin{array}{l}\text { the state after the input } \\ \text { is one level }\end{array} & \text { if } \tilde{u}(\psi) \neq u_{1} .\end{cases}$

As one can easily verify, INC changes the first " 0 " bit counting from the right to " 1 " and leave other bits untouched. A symmetric remark applies to the operation DEC. Therefore, bit $L$ (bit 1, resp.) is the most (least, resp.) important bit, in the sense that to switch bit $j$ from $0(1$, resp.) to $1(0$, resp.), one has to first switch all the lower bits to 1 ( 0 , resp.). Fig. 4 illustrates the INC and DEC operations for the case of $L=3$.

Given $u \in U$, the state transition function can be expressed as:

$$
\begin{aligned}
& \Xi_{\mathrm{d}}(\psi, u)= \\
& \begin{cases}\underbrace{\operatorname{INC} \circ \cdots \operatorname{INC}}_{n \text { INCs }}(\psi) & \text { if } u-\tilde{u}(\psi)=0, \tilde{u}(\psi)=n \delta_{u}, \\
\underbrace{\mathrm{DEC} \circ \cdots \mathrm{DEC}}_{n \mathrm{DEC} s}(\psi) & \text { if } u-\tilde{u}(\psi)=-n \delta_{u} .\end{cases}
\end{aligned}
$$

where "o" denotes composition of functions.

Proposition 3.2. Any state is reachable. Let $\psi_{i}, i=$ 1,2 , be two states. Let bit $n_{0}$ be the leftmost bit at which $\psi_{1}$ and $\psi_{2}$ differ, and let $n_{\mathrm{a}}$ be the number of alternating bit pairs in $\psi_{2}$ from bit $n_{0}$ through bit 1 . Then $\psi_{2}$ is reachable from $\psi_{1}$ by applying an input string $s_{\mathrm{a}}^{*} \in S_{\mathrm{A}}$ of length $n_{\mathrm{a}}+1$, and this is the shortest possible string length for achieving the transition.

The proposition is a straightforward consequence of the state transition map $\Xi_{\mathrm{d}}$.

Corollary 3.1. Any state is reachable from any other state with some $s_{\mathrm{a}}^{*} \in S_{\mathrm{A}}$ of length no more than $L$.

The following example illustrates Proposition 3.2 as well as how to actually construct the input string.

Example 3.1. Assume $L=5, \psi_{1}=00100, \psi_{2}=01011$. Then $n_{0}=4, n_{\mathrm{a}}=2$, and the alternating input string $s_{\mathrm{a}}^{*}$ for achieving the state transition has length 3 . The procedure for the state transition is as follows:

Step 0: $\psi_{1}$ contains one "1", so the current input value is $u_{2}$.

Step 1: Apply $u_{5}$ (3 consecutive INCs) to make bit 4 " 1 " and the state becomes 01111 .

Step 2: Apply $u_{2}$ (3 consecutive DECs) to make bit 3 " 0 " and the state becomes 01000 .

Step 3: Apply $u_{4}$ (2 consecutive INCs) to get $\psi_{2}$.

\section{A state-space reduction scheme}

\subsection{Reduction of the state space}

In general, one needs to store the output values of $2^{L}$ states for the value inversion problem. For a reasonable discretization level $L$, this may take lots of memory. In addition, computation cost for sorting and searching these states will be very high. Therefore, reducing the number of states without compromising control accuracy is of practical interest.

Two states $\psi_{1}, \psi_{2} \in \Psi_{\mathrm{d}}$ are equivalent, denoted as $\psi_{1} \equiv \psi_{2}$, if

$\Gamma\left[s, \psi_{1}\right]=\Gamma\left[s, \psi_{2}\right], \forall s \in S$.

We call a hysteron in the discretized Preisach operator non-trivial if its associated weight is not zero, and trivial otherwise. Existence of trivial hysterons leads to equivalent states. This is illustrated in Fig. 5(a), where the hysterons marked with "•" (and labeled by $\left.\gamma_{1}, \ldots, \gamma_{5}\right)$ are assumed to be non-trivial and those 


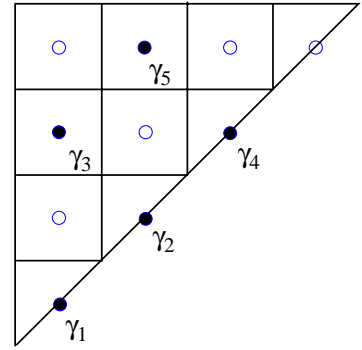

(a)

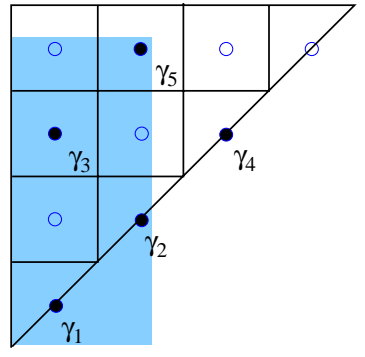

(b)
Fig. 5. (a) Illustration of equivalent states $(L=4)$, (b) illustration of the shaded set.

marked with "o" are assumed to be trivial. It is easy to verify that the following states in Fig. 5(a) are equivalent: 0101, 0110, 1001 and 1010. From the experimental result of density identification (see Fig. 8), indeed many hysterons carry weights of zero or close to zero, and this provides room for the state-space reduction.

The original state space $\Psi_{\mathrm{d}}$ is thus a disjoint union of equivalence classes of states. $\Psi_{\mathrm{d}}$ can be reduced, so that in the reduced state space $\widetilde{\Psi}$ each element is an equivalence class in $\Psi_{\mathrm{d}}$, i.e., $\widetilde{\Psi}=\Psi_{\mathrm{d}} / \equiv$. Denote the set of non-trivial hysterons as $\mathscr{N}$, i.e., $\mathscr{N} \triangleq\left\{\hat{\gamma}_{\beta, \alpha}\right.$ : $\left.v_{\beta, \alpha}>0\right\}$, where $v_{\beta, \alpha}$ is the weight of $\hat{\gamma}_{\beta, \alpha}$. For $\psi \in \Psi_{\mathrm{d}}$, define $\mathscr{S}(\psi)$ to be the set of non-trivial hysterons underneath the memory curve corresponding to $\psi$. From the example above, one can see that $\psi_{1} \equiv \psi_{2}$ if and only if $\mathscr{S}\left(\psi_{1}\right)=\mathscr{S}\left(\psi_{2}\right)$. Therefore, a member of $\widetilde{\Psi}$ can be identified with a subset $\tilde{\psi}$ of $\mathscr{N}$ that satisfies the following condition: there exists $\psi \in \Psi_{\mathrm{d}}$, such that $\tilde{\psi}=\mathscr{S}(\psi)$. To better capture the latter property, we introduce the notion of a lower-left-shaded set. The lower-left-shaded set (abbreviated as the shaded set hereafter) $\mathscr{A}\left(\hat{\gamma}_{\beta, \alpha}\right)$ of a hysteron $\hat{\gamma}_{\beta, \alpha} \in \mathscr{N}$ is defined to be

$$
\begin{aligned}
\mathscr{A}\left(\hat{\gamma}_{\beta, \alpha}\right) & =\left\{\hat{\gamma}_{\beta^{\prime}, \alpha^{\prime}} \in \mathscr{N}: \hat{\gamma}_{\beta^{\prime}, \alpha^{\prime}}\right. \\
& \left.\neq \hat{\gamma}_{\beta, \alpha}, \beta^{\prime} \leqslant \beta, \alpha^{\prime} \leqslant \alpha\right\} .
\end{aligned}
$$

The geometric interpretation of the shaded set of $\hat{\gamma}_{\beta, \alpha}$ is clear: imagining two rays from $\hat{\gamma}_{\beta, \alpha}$ in the Preisach plane, one pointing downwards and the other to the left, the shaded set consists of non-trivial hysterons lying between the two rays. For example, in Fig. 5(b), $\mathscr{A}\left(\gamma_{5}\right)=\left\{\gamma_{1}, \gamma_{2}, \gamma_{3}\right\}$. If $\hat{\gamma}_{\beta, \alpha}$ lies underneath some memory curve $\psi^{\prime}$, it follows that all elements of $\mathscr{A}\left(\hat{\gamma}_{\beta, \alpha}\right)$ must also lie underneath $\psi^{\prime}$. Therefore, $\tilde{\psi} \subset \mathscr{N}$ is identified with a member of $\widetilde{\Psi}$ if and only if the following holds:

$$
\mathscr{A}\left(\hat{\gamma}_{\beta, \alpha}\right) \subset \tilde{\psi}, \forall \hat{\gamma}_{\beta, \alpha} \in \tilde{\psi} .
$$

To ease presentation, from now on we will simply write $\tilde{\psi} \in \widetilde{\Psi}$ if (3) is satisfied. One can now list all members in $\widetilde{\Psi}$ using a tree-structured algorithm:

Step 1: List the equivalence class having no nontrivial hysterons (negative saturation).

Step 2: List equivalence classes with one constituent non-trivial hysteron, i.e., the shaded set of every such hysteron is empty.

Step 3: Starting from each equivalence class (parent class) $\tilde{\psi}$ with $n$ non-trivial hysterons, list equivalence classes (children classes) with $n+1$ non-trivial hysterons by finding another hysteron $\hat{\gamma} \in \mathscr{N}$ such that:

$\circ \hat{\gamma}$ is not included in $\tilde{\psi}$,

$\circ \mathscr{A}(\hat{\gamma}) \subset \tilde{\psi}$, i.e., $\tilde{\psi} \cup \hat{\gamma}$ is an eligible member of $\widetilde{\Psi}$, and

○ $\tilde{\psi} \cup \hat{\gamma}$ does not coincide with any other equivalence class $\tilde{\psi}^{\prime}$ with $n+1$ constituent hysterons that has been listed so far.

Step 4: Continue Step 3 until $\tilde{\psi}=\mathscr{N}$ (positive saturation) is listed.

The equivalence classes are sorted according to their output values during the above enumeration process. One can save computation time by using the fact that the output of a child class is always greater than that of its parent.

\subsection{Generation of best representative states}

For the purpose of realizing state transition, one needs to find a representative state $\psi \in \Psi_{\text {d }}$, i.e., a memory curve, for every $\tilde{\psi} \in \widetilde{\Psi}$. From Proposition 3.2 , the number of alternating bit pairs of a state $\psi$ is closely related to the number of input reversals required for the state transition. Therefore, the best representative state $\psi^{*} \in \Psi_{\mathrm{d}}$ for $\tilde{\psi} \in \widetilde{\Psi}$ should have the least number of alternating bit pairs among all states in the equivalence class $\tilde{\psi}$.

An algorithm is developed here to generate the optimal representative $\psi^{*}$ for $\tilde{\psi} \in \widetilde{\Psi}$. First draw two 


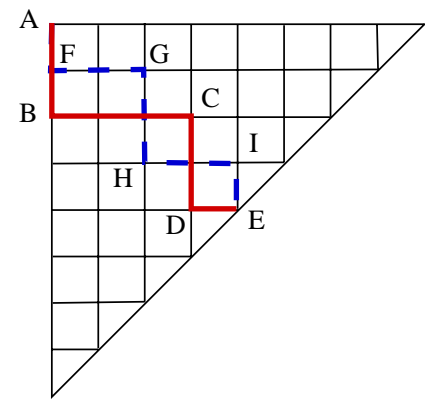

Fig. 6. Illustration of the proof of Proposition 4.1.

candidate memory curves $\psi_{\downarrow}^{*}$ and $\psi_{\rightarrow}^{*}$, and then pick $\psi^{*}$ to be the one whose number of alternating bit pairs is less. When growing a memory curve starting from the left upper corner of the discretized Preisach plane, one has two possible directions for each segment of the curve: going downwards (denoted as " $\downarrow$ ”) or going to the right (denoted as " $\rightarrow$ "). The candidate $\psi_{\downarrow}^{*}$ is obtained as follows: start with " $\downarrow$ " and continue that direction as long as it is feasible to do so (i.e., no constituent hysteron of $\tilde{\psi}$ is left out); when it is infeasible to continue " $\downarrow$ ", switch to " $\rightarrow$ " and keep going with that direction until it is infeasible for $\tilde{\psi}$ (i.e., nonconstituent hysterons will be included). Continue with these rules until all $L$ segments are drawn. Similarly one obtains $\psi_{\rightarrow}^{*}$ by starting with " $\rightarrow$ ". Note that “ $\rightarrow$ " is feasible whenever " $\downarrow$ " is not, and vice versa.

Proposition 4.1. The representative $\psi^{*}$ obtained in the above scheme has the least number of alternating bit pairs among all states in the equivalence class $\tilde{\psi}$.

Proof. For any state $\psi \in \tilde{\psi}$ starting with “ $\downarrow$ ”, one can show its number of alternating bit pairs is no less than that of $\psi_{\downarrow}^{*}$ by exploiting the strategy in generating $\psi_{\downarrow}^{*}$.

Instead of giving a general proof, we will illustrate the essential idea by looking at a concrete example with discretization level $L=8$ (Fig. 6). Assume that the memory curve represented by the bold-faced lines A-B-C-D-E ("00111001") is $\psi_{\downarrow}^{*}$. Let $\psi$ be any other state in the same equivalence class $\tilde{\psi}$ starting with “ $\downarrow$ ”. Now imagine we are growing the two curves $\psi_{\downarrow}^{*}$ and $\psi$ segment by segment, starting from the left upper corner. The curve $\psi$ has to switch to " $\rightarrow$ " no later than it reaches the point B (since otherwise it will be infeasible). This implies that when the first alternating bit pair in $\psi_{\rightarrow}^{*}$ occurs, at least one alternating bit pair has occurred in $\psi$. For the same reason, $\psi$ has to switch to " $\downarrow$ " before $\psi_{\downarrow}^{*}$ does so at point C. This argument goes on until the line $\alpha=\beta$ is hit and the drawing is completed. Hence, the number of alternating bit pairs in $\psi$ is no less than that in $\psi_{\downarrow}^{*}$. The curve represented by the dashed lines A-F-G-H-I-E in Fig. 6 gives an example of such $\psi$.

Analogously for any state $\psi$ starting with “ $\rightarrow$ ”, one can show its number of alternating bit pairs is no less than that of $\psi_{\rightarrow}^{*}$. The proof is now complete.

Example 4.1. For the equivalence class $\left\{\gamma_{1}, \gamma_{2}, \gamma_{3}\right\}$ in Fig. 5(a), $\psi_{\downarrow}^{*}=0110$ with two alternating bit pairs and $\psi_{\rightarrow}^{*}=1001$ with the same number of alternating bit pairs. So $\psi^{*}=\psi_{\downarrow}^{*}\left(\right.$ or $\left.\psi_{\rightarrow}^{*}\right)$.

\section{Experimental results}

In this section the value inversion approach together with the state-space reduction scheme is applied to micro-positioning control of a magnetostrictive actuator. Refer to [18] for a description of the experimental setup. The displacement output $y$ of the actuator is controlled by the magnetic field generated through the current $I$ in a coil. Fig. 7 displays the hysteresis between the displacement output and the current input in the actuator.

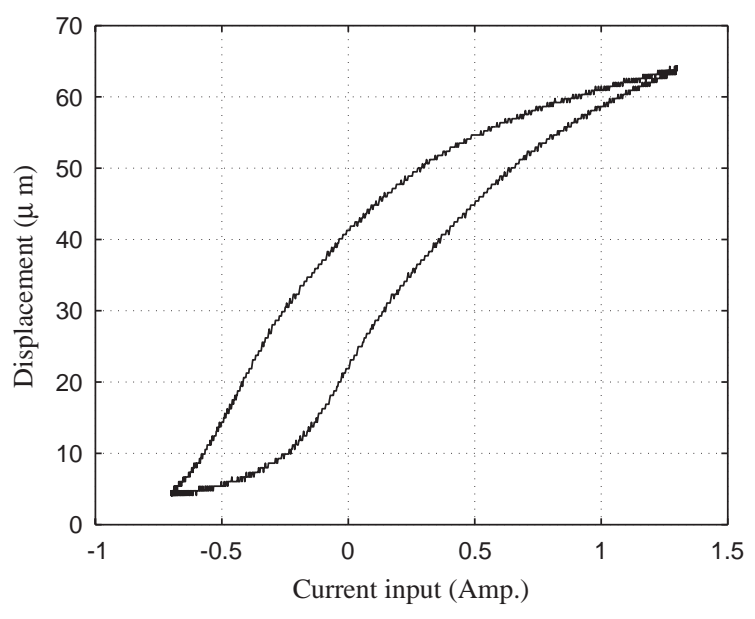

Fig. 7. Hysteresis in the magnetostrictive actuator. 


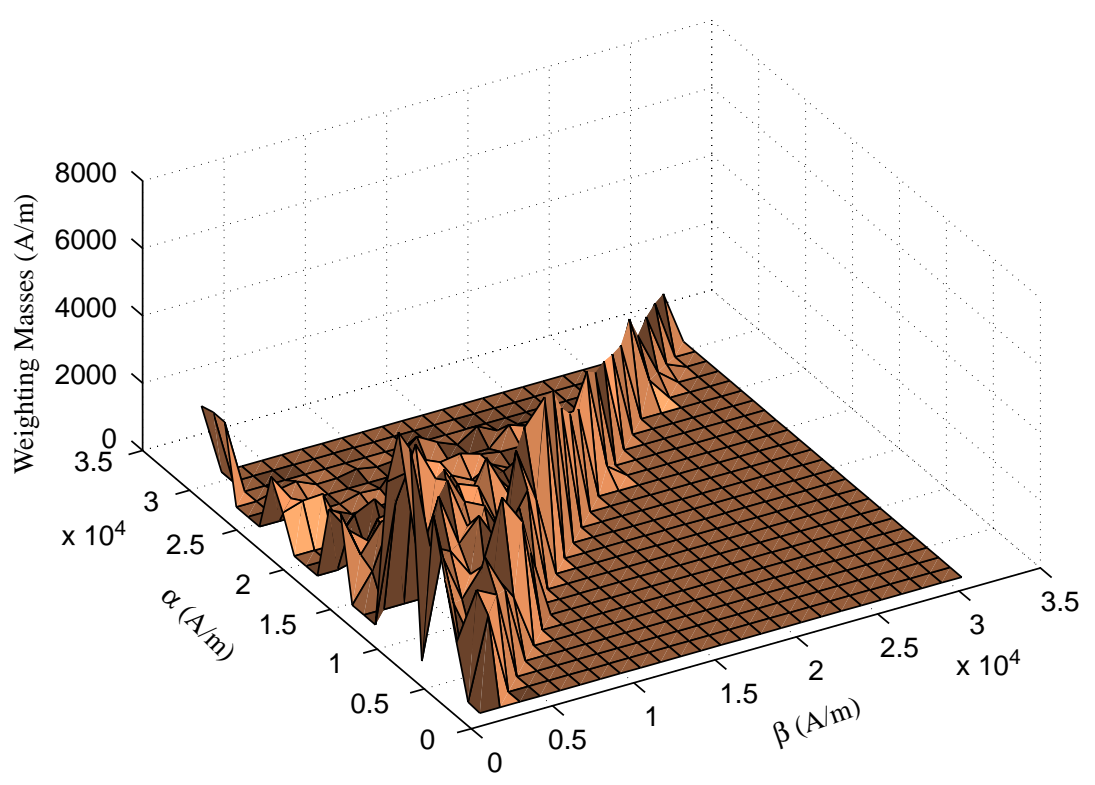

Fig. 8. Distribution of the Preisach weighting masses $(L=25)$.

When operated in a low-frequency range (typically below $5 \mathrm{~Hz}$ ), the displacement $y$ can be related to the bulk magnetization $M$ by a square law $\lambda=a_{1} M^{2}$ for some constant $a_{1}>0$, and the input current $I$ can be related to the magnetic field $H$ along the rod direction by $H=c_{0} I$, where $c_{0}$ is the coil factor [21]. Then the magnetostrictive hysteresis between $y$ and $I$ is fully captured by the ferromagnetic hysteresis between $M$ and $H$, which is modeled by the Preisach operator. The Preisach plane is discretized with $L=25$ which results in 300 hysterons. Fig. 8 displays the experimentally identified Preisach weighting masses. By treating 201 hysterons whose weights are zero or very small as trivial, we are left with 99 non-trivial hysterons. The final number of states in the reduced state space is 99,217 compared to $33,554,432$ in the original state space.

Given a sequence of eight desired displacement values $(10,30,15,40,20,40,60$ and $50 \mu \mathrm{m})$, the control objective is to drive the actuator head to these positions consecutively. Three control schemes are implemented to achieve the positioning goal. The first one is based on the value inversion scheme, the second is based on the closest match algorithm for trajectory inversion (see [19]), and the third scheme is based on a non-hysteretic model where the input-output
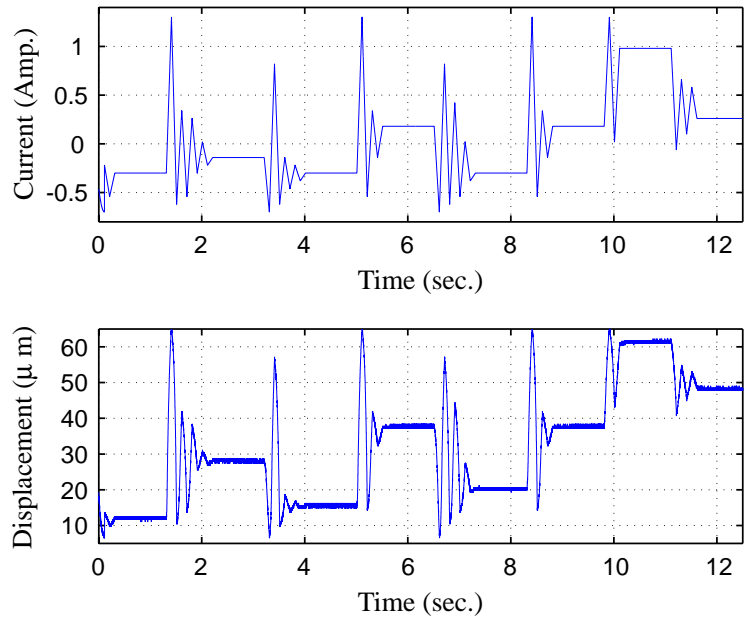

Fig. 9. Micro-positioning control based on the value inversion scheme.

relationship is approximated by a single-valued function $y=-7.44 I^{3}-2.63 I^{2}+40.81 I+30.34$. The trajectories of the current input and the measured displacement under these schemes are shown in Figs. 9-11. For presentation purposes, we intentionally hold the input current constant for about $1 \mathrm{~s}$ after each positioning is completed. Fig. 12 compares the 

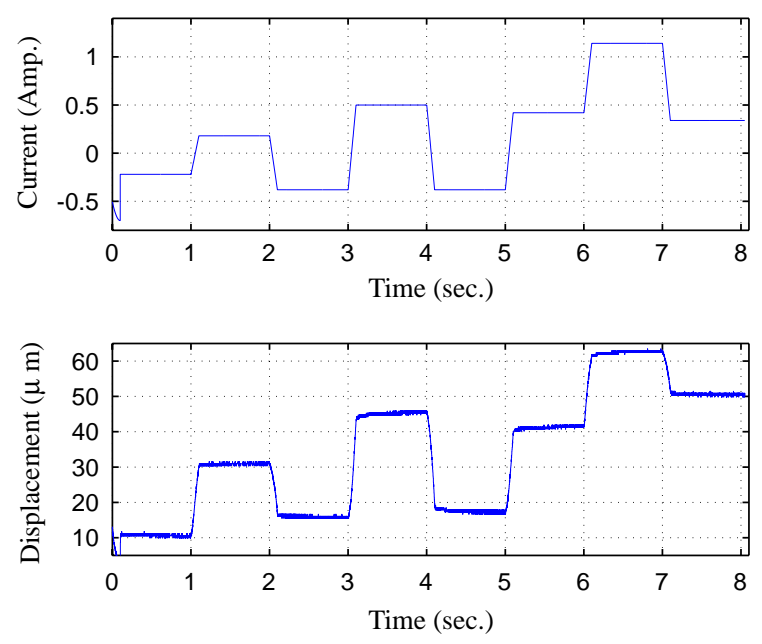

Fig. 10. Micro-positioning control based on the closest match algorithm.
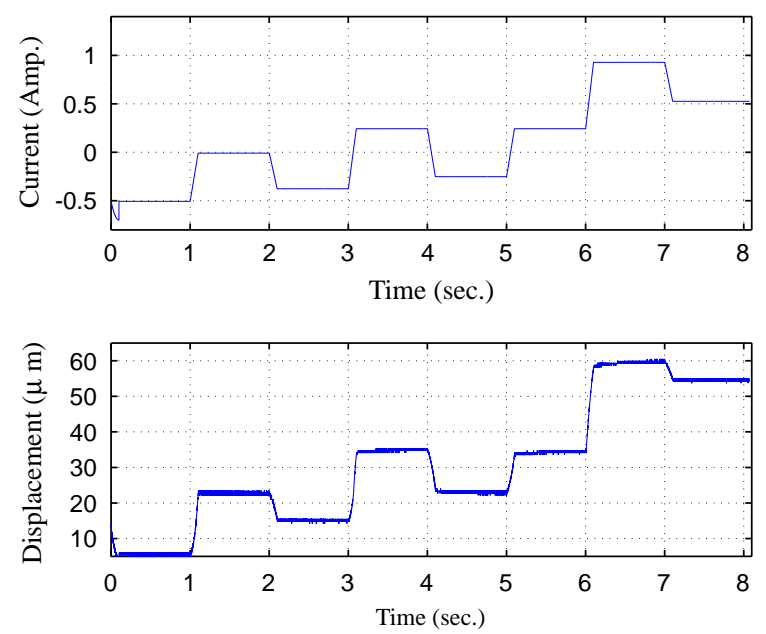

Fig. 11. Micro-positioning control based on a non-hysteretic model.

errors of the three schemes for the eight positioning tasks. It can be seen that Scheme 1 yields the minimum positioning error. As a trajectory inversion algorithm, Scheme 2 does not allow input reversals for each desired output value and thus has less control freedom than Scheme 1 does. This explains why Scheme 1 is better than Scheme 2. Scheme 3 delivers the worst performance because hysteresis is not taken into account.

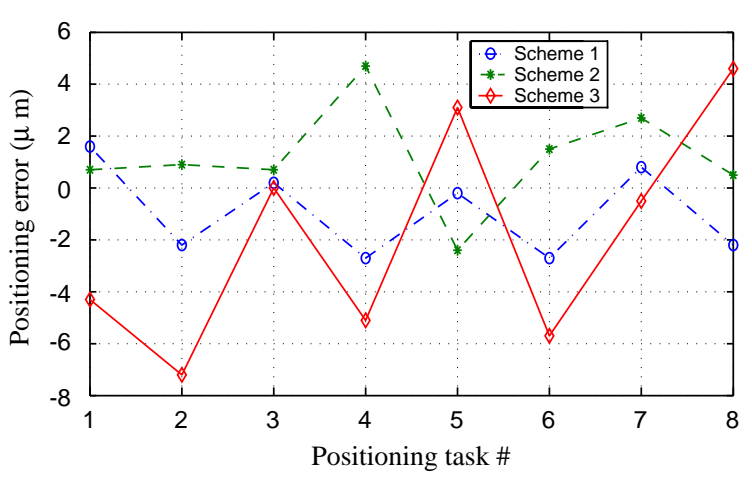

Fig. 12. Comparison of three schemes. Scheme 1: the value inversion algorithm; Scheme 2: the closest match algorithm; Scheme 3: the inversion algorithm based on a non-hysteretic model.

\section{Conclusions}

In this paper a novel type of inversion problem, called the value inversion problem, for a class of discretized hysteresis operators has been studied. Unlike most inversion problems discussed in the literature on hysteresis control, the value inversion problem is to find an optimal input trajectory given a desired value of the hysteresis output. This problem was motivated by positioning applications of smart actuators.

The Preisach operator has been used for the modeling of hysteresis. When discretized, it can be represented by an FSM. Based on a concise indexing scheme for the memory curve, the dynamics of the FSM is captured by simple rules. The original value inversion problem was converted to a state reachability problem of the FSM. Implementation of state transitions were illustrated through examples. The notion of state-space reduction was developed for a discretized Preisach operator, and algorithms for generating the reduced state space and for constructing the optimal representative state were also presented. This approach has been applied to micro-positioning control of a magnetostrictive actuator and its effectiveness has been demonstrated through comparison with two other inversion schemes.

\section{Acknowledgements}

This research was supported by the Army Research Office under the ODDR\&E MURI97 Program Grant 
no. DAAG55-97-1-0114 to the Center for Dynamics and Control of Smart Structures (through Harvard University).

\section{References}

[1] A.A. Adly, I.D. Mayeygoyz, A. Bergqvist, Preisach modeling of magnetostrictive hysteresis, J. Appl. Phys. 69 (8) (1991) 5777-5779.

[2] H.T. Banks, A.J. Kurdila, G. Webb, Identification of hysteretic control influence operators representing smart actuators, Part I: formulation, Math. Problems Eng. 3 (4) (1997) 287-328.

[3] L.S. Bobrow, M.A. Arbib, Discrete Mathematics: Applied Algebra for Computer and Information Science, W.B. Saunders Company, 1974.

[4] M. Brokate, J. Sprekels, Hysteresis and Phase Transitions, Springer, New York, 1996.

[5] D. Croft, G. Shed, S. Devasia, Creep, hysteresis, and vibration compensation for piezoactuators: atomic force microscopy application, J. Dyn. Systems Meas. Control 123 (1) (2001) $35-43$.

[6] W.S. Galinaitis, R.C. Rogers, Control of a hysteretic actuator using inverse hysteresis compensation, in: V. Varadan (Ed.), Mathematics and Control in Smart Structures, SPIE, vol. 3323, 1998, pp. 267-277.

[7] P. Ge, M. Jouaneh, Tracking control of a piezoceramic actuator, IEEE Trans. Control Systems Technol. 4 (3) (1996) 209-216.

[8] R.B. Gorbet, D.W.L. Wang, K.A. Morris, Preisach model identification of a two-wire SMA actuator, in: Proceedings of IEEE International Conference on Robotics and Automation, 1998, pp. 2161-2167.

[9] R.B. Gorbet, K.A. Morris, D.W.L. Wang, Control of hysteretic systems: a state-space approach, in: Y. Yamamoto, S. Hara (Eds.), Learning, Control and Hybrid Systems, Lecture Notes in Control and Information Sciences, vol. 241, Springer, New York, 1998, pp. 432-451.
[10] D. Hughes, J.T. Wen, Preisach modeling and compensation for smart material hysteresis, in: G.L. Anderson, D.C. Lagoudar (Eds.), Active Materials and Smart Structures, SPIE, vol. 2427, (1994) pp. 50-64.

[11] I.D. Mayergoyz, Mathematical Models of Hysteresis, Springer, Berlin, 1991.

[12] S.O.R. Moheimani, G.C. Goodwin, Guest editorial introduction to the special issue on dynamics and control of smart structures, IEEE Trans. Control System Technol. 9 (1) (2001) 3-4.

[13] C. Natale, F. Velardi, C. Visone, Identification and compensation of Preisach hysteresis models for magnetostrictive actuators, Physica B 306 (2001) 161-165.

[14] H. Perez, S. Devasia, Optimal output-transitions for linear systems, Automatica 39 (2) (2003) 181-192.

[15] J. Schäfer, H. Janocha, Compensation of hysteresis in solidstate actuators, Sensors Actuators A 49 (1-2) (1995) 97-102.

[16] R.C. Smith, Inverse compensation for hysteresis in magnetostrictive transducers, CRSC Technical Report, North Carolina State University (CRSC-TR98-36).

[17] X. Tan, Control of smart actuators, Ph.D. Thesis, University of Maryland, available as ISR Technical Report PhD 2002-8 at http://techreports.isr.umd.edu/ARCHIVE (2002).

[18] X. Tan, J.S. Baras, Modeling and control of hysteresis in magnetostrictive actuators, Automatica 40 (9) (2004) $1469-1480$.

[19] X. Tan, R. Venkataraman, P.S. Krishnaprasad, Control of hysteresis: theory and experimental results, in: V.S. Rao (Ed.), Modeling, Signal Processing, and Control in Smart Structures, SPIE, vol. 4326, 2001, pp. 101-112.

[20] G. Tao, P.V. Kokotović, Adaptive control of plants with unknown hystereses, IEEE Trans. Automat. Control 40 (2) (1995) 200-212.

[21] R. Venkataraman, Modeling and adaptive control of magnetostrictive actuators, Ph.D. Thesis, University of Maryland, College Park, 1999.

[22] A. Visintin, Differential Models of Hysteresis, Springer, Berlin, 1994. 